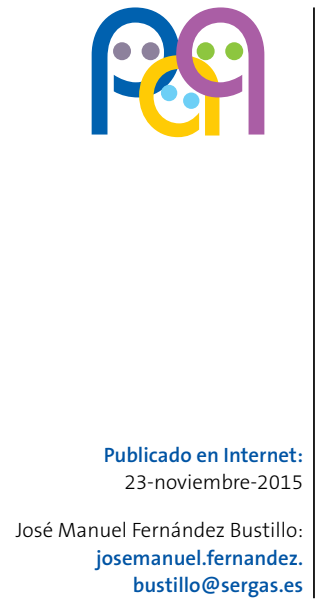

Palabras clave:

- Sobrepeso

- Obesidad

- Prevalencia

- Crecimiento

\section{Sobrepeso y obesidad: ¿cuál es nuestra realidad?, ¿qué referencia utilizamos? El estudio OBESGAL}

\author{
J. M. Fernández Bustillo ${ }^{a}$, P. Pereira García ${ }^{b}$, M. J. Méndez Busteloc, \\ Grupo Colaborativo Obesgal ${ }^{d}$
}

aPediatra. CS Bertamiráns. Ames, A Coruña. España • bPediatra. CS Milladoiro. Ames, A Coruña. España • 'Pediatra. CS Oroso. Ames, A Coruña. España • dG. Antelo Rodríguez, C. Belo González, M. T. Calvo Lorenzo, M. F. Crespo Vázquez, I. Díaz-Cardama Sousa, C. A. Díaz Sánchez, J. M. Fernández Bustillo, M. Fernández Pérez, A. Fernández Pombo, S. Garabal Sánchez,

C. García Cendón, C. Hernández Valencia, M. J. Méndez Bustelo, M. E. Noya Beiroa, J. Pena Nieto, P. Pereira García, M. C. Pérez Arias, M. Picón Cotos, M. J. Prado Bustamante, M. Pumarega Vergara, M. T. Ruíz Díaz, J. Sangabriel Villar, F. Vázquez Couso, A. Villanueva Jeremías.

Introducción: la obesidad, en la edad pediátrica, constituye un desafío de salud pública. Por este motivo, llevamos a cabo un estudio comparativo de los resultados obtenidos en el momento actual en nuestra área sanitaria, para valorar el estado de nutrición de la población infantil de 2 a 15 años, atendidos en distintos centros de Atención Primaria, estudiando la prevalencia de sobrepeso, obesidad y obesidad mórbida en nuestra comunidad autónoma y confrontarlos con estudios previos a nivel nacional.

Material y métodos: la población del estudio está compuesta de una muestra representativa de 15142 niños, de edades comprendidas entre 2 y 15 años. Se revisaron las historias clínicas y se recogieron los datos antropométricos (peso, talla e índice de masa corporal [IMC]) procedentes de los controles establecidos en el Programa de Salud Infantil (Programa do Neno San), obteniendo una muestra final de 12643 individuos (6433 [50,9\%] niños y 6210 niñas [49,1\%]).

La prevalencia de sobrepeso y obesidad se analizó en función de las variables edad y sexo, estableciéndose como puntos de corte los correspondientes a los percentiles p85 (sobrepeso) y p95 (obesidad). Para categorizar el IMC se utilizaron las gráficas para edad y sexo del estudio longitudinal de crecimiento de la Fundación Orbegozo que figuran en la historia clínica del SERGAS (Servizo Galego de Saúde). Las estimaciones de prevalencia se acompañaron de los respectivos intervalos de confianza del $95 \%$ (IC 95). Para la comparación de variables cualitativas se utilizó la prueba 2. Los datos se analizaron con el paquete estadístico SPSS $®$ para Windows ${ }^{\circledR}$, versión 12.0 .

Comparamos nuestros resultados con los de estudios previos: el estudio enKid (1988-2000), y el estudio Aladino (2010-2011).

Resultados: la prevalencia de sobrepeso $(9,3 \%)$ y obesidad $(9,7 \%)$ en nuestro estudio es inferior, globalmente, a la obtenida en otros estudios de referencia nacionales: enKid (12,4/13,9\%), Aladino $(26,2 / 18,3 \%)$, etc., aunque no es fácil establecer comparaciones puesto que los criterios empleados para definir sobrepeso y obesidad no son los mismos, no usan las mismas tablas como referencia ni el mismo punto de corte.

Conclusiones: la prevalencia de sobrepeso y obesidad varía según sea el estudio de referencia utilizado alejándose, en nuestro caso, de la cifra "oficial" esperada. Es necesario consensuar y unificar criterios (Organización Mundial de la Salud [OMS], International Obesity TaskForce [IOTF], Orbegozo, etc.) para establecer el diagnóstico de sobrepeso u obesidad a partir del IMC. 


\section{Overweight and obesity: what is our reality?, what reference do we use? The OBESGAL study}

Introduction: obesity in children is a challenge for public health. Therefore, we conducted a comparative study of the results obtained in the present moment in our health area, to assess the nutritional status of children 2-15 years old who presented in different primary care centers, studying the prevalence of overweight, obesity and morbid obesity in our region and comparing them with previous studies nationwide.

Subjects and method: the study population is composed of a representative sample of 15142 children, aged 2 to 15 years old. The medical records were reviewed and anthropometric data (weight, height and $\mathrm{BMI}$ ) were collected from the visits of the Well Child Program (Program do Neno San) to obtain a final sample of 12643 individuals [6433 boys (50.9\%) and 6210 girls (49.1\%)].

The prevalence of overweight and obesity was analyzed according to age and sex variables, establishing a cutoff corresponding to the p85 percentile (overweight) and p95 (obesity). To categorize the BMI, charts for age and sex of the longitudinal growth study Orbegozo Foundation, contained in the SERGAS medical record (Galician Health), were used. Prevalence estimates were accompanied by the respective $95 \%$ confidence intervals $(\mathrm{Cl})$. Chi square test was used to compare qualitative variables. The data were analyzed using the statistical package SPSS for Windows, version 12.0.

We compared our results with those of previous studies: the enKid study (1988-2000), and Aladino study (2010-2011).

Results: the prevalence of overweight $(9.3 \%)$ and obesity $(9.7 \%)$ in our study is lower, overall, than that obtained in other studies of national reference: enKid (12.4 / 13.9\%), Aladino (26.2 / 18.3\%), etc., although it is difficult to make comparisons because the criteria used to define overweight and obesity are not the same, we do not use the same charts as a reference or the same cutoff.

Key words:

- Overweight

- Obesity

- Prevalence

Conclusions: the prevalence of overweight and obesity varies depending on the baseline study used, being in our case far from the "official" expected figure. It requires consensus and unified criteria

- Growth (WHO, IOTF, Orbegozo, etc.) to establish the diagnosis of overweight or obesity using the BMI.

\section{INTRODUCCIÓN}

En las últimas décadas la obesidad, tanto en los países desarrollados como en vías de desarrollo, ha experimentado un notable incremento y por tal motivo la 57. a Asamblea Mundial de la Salud, en mayo de 2004, la declara como la "epidemia del siglo XXI" y aprueba la creación de una estrategia sobre nutrición, actividad física, obesidad y salud $(\mathrm{NAOS})^{1}$, que el Ministerio de Sanidad desarrolla a partir del $2005^{2}$

En la edad pediátrica la obesidad es un problema generalizado, convirtiéndose en una epidemia silenciosa que constituye un verdadero desafío de salud pública, ya que según cálculos estimativos de la $\mathrm{OMS}^{3}$ más de 40 millones de niños menores de cinco años tenían sobrepeso en 2011 y la prevalencia se elevará al $11 \%$ a nivel mundial para el año 2025, 70 millones de niños, si continúan las tendencias actuales ${ }^{4}$.
Los malos hábitos alimentarios, con una gran oferta de nutrientes hipercalóricos, y los cambios en los estilos de vida (sedentarismo, etc.), unidos a la cada vez más evidente base genética, parecen constituir los factores de riesgo para el desarrollo de la obesidad infantil ${ }^{5}$, lo que induce un notable impacto en la calidad de vida futura (morbimortalidad) y en el gasto sanitario. Del mismo modo, se percibe un notable incremento de la obesidad grave en la población infantil ${ }^{6}$, con graves consecuencias a corto y largo plazo para la salud cardiovascular, metabólica, etc., por lo que la prevención constituye una prioridad sanitaria global ya que el niño obeso tiene un elevado riesgo de obesidad en la vida adulta ${ }^{7}$.

Nuestro objetivo es valorar el estado de nutrición de la población infantil de 2 a 14 años atendidos en distintos centros de Atención Primaria, estudiando la prevalencia de sobrepeso y obesidad mórbida en nuestra comunidad. 


\section{MATERIAL Y MÉTODOS}

Se trata de un estudio descriptivo, retrospectivo y transversal, de junio a septiembre de 2013. Se revisaron las historias clínicas de 15142 niños/as de la población gallega, pertenecientes a los municipios de Ames, A Estrada, Lousame, Lugo, Órdenes, Oroso, Santiago de Compostela y Tomiño, y se recogieron los datos procedentes de los controles de salud establecidos en el Programa de Salud Infantil (Programa do Neno San) ${ }^{8}$. El Servicio Galego de Saúde (SERGAS) dispone de un sistema de historia clínica informatizada en la totalidad de sus centros $y$, dada la altísima cobertura del programa del niño sano, los datos obtenidos nos dan un reflejo de la situación a nivel de la comunidad autónoma.

Todas las mediciones fueron realizadas por personal cualificado (enfermeras pediátricas) en las consultas de Pediatría de los centros de salud anteriormente referidos. Las valoraciones de peso y talla fueron realizadas en ropa interior y descalzos, con básculas calibradas y una precisión de 100 gramos y la talla con un tallímetro rígido inextensible de pared con un intervalo de precisión de $0,1 \mathrm{~cm}$ con los pacientes en bipedestación, descalzos y con la cabeza situada en el plano de Frankfurt.

Cada uno de los participantes en el estudio recogió, en una hoja Excel ${ }^{\oplus}$, los siguientes campos: fecha de nacimiento, sexo, peso, talla, así como la fecha de su realización, obteniendo una muestra final de 12643 individuos (6433 niños [50,9\%] y 6210 niñas [49,1\%]).

EI IMC (peso en kilogramos/talla al cuadrado en metros) se calculó automáticamente en la misma hoja Excel ${ }^{\oplus}$. Para categorizar el IMC se utilizaron las gráficas para la edad y sexo del estudio longitudinal de crecimiento de la Fundación Orbegozo que figuran en la historia clínica del SERGAS ${ }^{9}$.

Una vez recogidos los datos se estratificaron en tres grupos, siguiendo la distribución etaria establecida por el SERGAS:

- Niños de 2-4 años: 3726 (29,5\%).

- Niños de 5-9 años: 5293 (41,9\%).

- Niños de 10-14 años: 3614 (28,6\%).
La prevalencia de sobrepeso y obesidad se analizó en función de las variables edad y sexo, estableciéndose como puntos de corte los correspondientes a los percentiles p85 (sobrepeso) y p95 (obesidad).

Para el cálculo de la prevalencia de la obesidad grave se utilizó como punto de corte el percentil 99 mediante la fórmula $120 \%$ del percentil 95 (1,2 $\times$ percentil 95) del IMC para la edad ${ }^{6}$, que es similar a los datos del percentil 99 de la gráfica de crecimiento.

Las estimaciones de prevalencia se acompañaron de los respectivos IC 95. Para la comparación de las variables cualitativas se utilizó la prueba $\bigoplus^{2}$. Los datos se analizaron con el paquete estadístico SPSS $^{\oplus}$ para Windows ${ }^{\circledR}$, versión 12.0 .

\section{RESULTADOS}

En nuestro estudio (Tabla 1) encontramos una prevalencia global de obesidad del 9,7\% y de sobrepeso del $9,3 \%$, alcanzando el $19 \%$ si analizamos conjuntamente la sobrecarga ponderal (sobrepeso más obesidad), siendo el grupo entre 10-14 años el que obtiene cifras más elevadas (11,2\% sobrepeso y el $11,9 \%$ obesidad). Si examinamos los grupos por separado, podemos ver que la sobrecarga ponderal aumenta progresivamente con la edad desde un $13 \%$ en el grupo de $2-4$ años hasta el $23,1 \%$ en el grupo de 10-14 años.

Si desglosamos el cómputo total por sexo y por edad (Tabla 1) podemos subrayar que existen diferencias por grupo de edad, tanto en las cifras totales como las parciales. Así en los varones de entre 5-9 años es cuando se alcanzan los índices más elevados para la obesidad, el 13,8\%, que asciende al $24 \%$ si valoramos conjuntamente el sobrepeso y la obesidad y esta tendencia se mantiene en el grupo de 10-14 años, a expensas del sobrepeso. En las niñas, es el grupo entre 10-14 años las que alcanzan cifras más elevadas tanto de sobrepeso $(9,8 \%)$ como de obesidad (11,7\%), siendo la prevalencia en obesidad similar a la de los niños para este grupo de edad (12\%). 
Cuando analizamos la tendencia de la obesidad, los datos muestran que a todas las edades la prevalencia de obesidad es menor en las niñas que en los varones.

En cuanto a los niños con obesidad grave (Tabla 1), existen diferencias notorias por grupo de edad y sexo, estando más acentuada en los niños varones (72,3\% del total de casos con obesidad grave) que en las niñas $(27,7 \%)$, siendo este grado de adiposidad más perceptible en el grupo de entre 5-9 años ya que supone el $49,7 \%$ del cómputo total, y que disminuye hasta el 35,5\% entre los 10-14 años; alcanzado no obstante cifras del $14,8 \%$ en edades tempranas (2-4 años).

\section{DISCUSIÓN}

La obesidad, considerada como la "epidemia del siglo XXI", ha experimentado un notable aumento en las últimas décadas, habiéndose realizado diferentes estudios en nuestro país que muestran este incremento. Así la prevalencia de obesidad infantil pasó del 4,9\% en 1984, según el estudio Paidos ${ }^{11}$, al 13,9\% entre 1988-2000, como refiere el estudio enKid ${ }^{12}$.

No obstante, la encuesta de salud poblacional elaborada por el Ministerio de Sanidad y Consumo, entre los años 2006-2007 en niños de 2-15 años ${ }^{13}$ encuentra una prevalencia global del $10,3 \%$ tomando como referencia el IMC recomendado por la IOTF, cifras que descendieron al 9,6\% en la encuesta realizada entre los años 2011-2012.

Durante el curso escolar 2010/2011, la Agencia Española de Seguridad Alimentaria y Nutrición (AESAN [ahora AECOSAN]) realizó el estudio Aladino (Alimentación, Actividad Física, Desarrollo infantil y Obesidad) con el objetivo de estimar la prevalencia de la obesidad infantil en niños y niñas de 6 a 9,9 años. Tomando como referencia los estándares de la OMS, se estimó una prevalencia de obesidad de un $20,9 \%$ en niños y $15,5 \%$ en niñas, alcanzando el $44,5 \%$ la prevalencia de sobrepeso, incluida la obesidad, en toda la población del estudio, cifras muy superiores a las encontradas por nosotros (Tabla 1).
La prevalencia de obesidad en nuestro estudio también es inferior a la del estudio nacional de referencia enKid, que utilizó los percentiles 85 y 97 de las tablas de Orbegozo de 1988 para definir sobrepeso y obesidad. Así, el estudio enKid refiere, para una población entre 2-24 años, una prevalencia de sobrepeso y obesidad para la zona norte (Galicia, Asturias, Cantabria, País Vasco, Navarra y La Rioja) del $12,7 \%$ y $12,3 \%$ respectivamente. Si nos referimos a edades pediátricas exclusivamente, se observa que la prevalencia, entre los 10-13 años, alcanza en el estudio enKid, las cifras más elevadas en cuanto al sobrepeso (14,6\%) y obesidad (16,6\%), elevándose en los niños hasta el 20,0\% y 21,9\% respectivamente, lo que concuerda con lo observado en nuestro estudio, pero con cifras sensiblemente inferiores en nuestra investigación, tanto globalmente $(12,2 \%$ obesidad y $11,3 \%$ sobrepeso) como por sexo. Hay que destacar que en nuestro estudio la prevalencia de obesidad es mayor en las niñas que en los niños en este grupo de edad, mientras que en edades inferiores es mayor en varones. Esto también fue observado en estudios como el de Canarias $^{14}$, mientras que en otros como en el de $\mathrm{Na-}$ varra la prevalencia es mayor en varones en estas edades ${ }^{15}$.

En la actualidad se insinúa que la tasa de obesidad entre niños y adolescentes se ha desacelerado ${ }^{16}, y$ que la prevalencia global, posiblemente, ha comenzado a estabilizarse en los últimos años ${ }^{17}$. Así se ratifica en el estudio Aladino, realizado en el año 2013, en el que se observa disminución de las prevalencias de sobrepeso y obesidad con respecto a las halladas previamente ${ }^{18}$. Sin embargo, una tendencia preocupante ha surgido en forma de obesidad infantil grave, aumentando progresivamente en la población infantojuvenil, alcanzando cifras del 4-6\% de todos los jóvenes de EE. UU. entre los 6-19 años, lo que implica graves consecuencias para la salud, con mayor riesgo de enfermedad cardiovascular, diabetes mellitus tipo 2 y muerte prematura en la edad adulta ${ }^{19}$. En nuestro estudio esta cifra es sensiblemente inferior, con tan solo 141 niños, lo que supone el 1,1\% del total, lo que concuerda con un estudio reciente realizado en Navarra que, utilizando como referencia los estándares de la 


\begin{tabular}{|c|c|c|c|}
\hline \multicolumn{4}{|c|}{ Sobrepeso y obesidad por grupo de edad } \\
\hline Edad (años) & Sobrepeso (>p85) & Obesidad (>p95) & Sobrepeso más obesidad \\
\hline $2-4$ & $6,4 \%$ & $6,5 \%$ & $13,0 \%$ \\
\hline $5-9$ & $10 \%$ & $10,4 \%$ & $20,4 \%$ \\
\hline $10-14$ & $11,2 \%$ & $11,9 \%$ & $23,1 \%$ \\
\hline Total & $9,3 \%$ & $9,7 \%$ & $19,0 \%$ \\
\hline \multicolumn{4}{|c|}{ Sobrepeso y obesidad por grupo de edad y sexo } \\
\hline Niños (edad) & Sobrepeso (>p85) & Obesidad (>p95) & Sobrepeso más obesidad \\
\hline 2-4 años & $6,8 \%$ & $6,7 \%$ & $14,5 \%$ \\
\hline 5-9 años & $10,2 \%$ & $13,8 \%$ & $24,0 \%$ \\
\hline 10-14 años & $12,5 \%$ & $12,0 \%$ & $24,5 \%$ \\
\hline Total & $9,8 \%$ & $11,5 \%$ & $21,4 \%$ \\
\hline Niñas (edad) & Sobrepeso (>p85) & Obesidad (>p95) & Sobrepeso más obesidad \\
\hline 2-4 años & $6,1 \%$ & $5,2 \%$ & $11,4 \%$ \\
\hline 5-9 años & $9,0 \%$ & $6,9 \%$ & $16,7 \%$ \\
\hline 10-14 años & $9,8 \%$ & $11,7 \%$ & $21,6 \%$ \\
\hline Total & $8,7 \%$ & $7,8 \%$ & $16,5 \%$ \\
\hline \multicolumn{4}{|c|}{ Obesidad grave (1,2 × IMC >p95): 141 niños } \\
\hline Grupo & Niños & Niñas & Total \\
\hline 2-4 años & 16 & 5 & $21(14,8 \%)$ \\
\hline 5-9 años & 55 & 15 & $70(49,7 \%)$ \\
\hline 10-14 años & 31 & 19 & $50(35,5 \%)$ \\
\hline Total & $102(72,3 \%)$ & $39(27,7 \%)$ & $141(100 \%)$ \\
\hline
\end{tabular}

IMC: índice de masa corporal.

OMS, encontró una prevalencia global de obesidad y obesidad grave similar a la nuestra (7,9\% y $1,2 \%)$, pero una prevalencia de sobrepeso muy superior a la nuestra $(21 \%)^{20}$. Sin embargo, es significativo que un porcentaje elevado del mismo, el $72,3 \%$, son varones, representando, en ambos sexos, la cifra más significativa, el 49,7\%, el grupo etario de 5-9 años, disminuyendo ligeramente entre los 1014 años. No obstante, este problema se inicia ya a edades tempranas, aunque con cifras sensiblemente inferiores, y el $14,8 \%$ de los niños/as con obesidad grave tienen 2-4 años.

La valoración de la obesidad comporta amplias dificultades metodológicas, al no existir consenso acerca de los puntos de corte a la hora de definirla, ya que, al utilizar distintas tablas de referencia, los valores de prevalencia son también diferentes. Para confrontar los resultados de este estudio con los ya existentes se valoró la situación ponderal teniendo en cuenta las curvas de crecimiento de la Fundación Orbegozo (FO $)^{21}$, los criterios de la
Internacional Obesity Task Force (IOTF) ${ }^{22}$ y las de la OMS $^{23}$ (Tabla 2).

La prevalencia de obesidad y sobrepeso en nuestra comunidad presenta unas cifras inferiores a las de otras zonas, sin embargo, observamos una obesidad grave más frecuente en varones en todas las edades.

La sensibilización de la población ante el problema de la obesidad infantil es fundamental para evitar su avance ya que solo el cambio en los estilos de vida la puede mejorar. La Atención Primaria tiene un papel esencial en el diagnóstico de control y prevención de las alteraciones de la nutrición. El programa de salud infantil vigila al niño desde el nacimiento hasta los 14 años, lo que nos permite detectar precozmente la obesidad infantil y nos da la posibilidad de actuar tempranamente. Así se ha visto que el tratamiento más exitoso en la obesidad, antes de la adolescencia, puede que sea la intervención en preescolares con programas de visitas frecuentes ${ }^{24}$. 
Tabla 2. Prevalencia de sobrepeso y obesidad (12 643 niños) según el punto de corte utilizado

\begin{tabular}{|l|c|c|c|}
\hline \multicolumn{1}{|c|}{ Gráfica } & Sobrepeso & Obesidad & Sobrepeso más obesidad \\
\hline OMS & 21,8 & 13,5 & 35,3 \\
\hline IOTF & 19,2 & 5,95 & 25,2 \\
\hline FO 2011 & 17,2 & 3,2 & 20,4 \\
\hline FO 2004 & 9,3 & 9,7 & 19 \\
\hline FO 1988 & 8,05 & 12,1 & 20,15 \\
\hline
\end{tabular}

FO: Fundación Orbegozo; IOTF: International Obesity TaskForce; OMS: Organización Mundial de la Salud.

La prevención de la obesidad es un aspecto fundamental de la promoción de la salud, por lo que es imprescindible que se apliquen criterios diagnósticos consensuados que faciliten el contraste regional, internacional y secular de las cifras de sobrecarga ponderal en la infancia ${ }^{25}$.

\section{CONFLICTO DE INTERESES}

Los autores declaran no presentar conflictos de intereses en relación con la preparación y publicación de este artículo.

Este trabajo fue presentado parcialmente en forma de comunicación al LXIV Congreso de la Sociedad de Pediatría de Galicia 2013 y al LXV Congreso de la Sociedad de Pediatría de Galicia 2014. Recibió el 3. ${ }^{\text {er }}$ Premio a la Mejor Comunicación Oral en Medicina Pediátrica en el LXIV Congreso de la Socie-

\section{BIBLIOGRAFÍA}

1. Estrategia Mundial sobre Régimen Alimentario, Actividad Física y Salud. Ginebra: OMS; 2004.

2. Agencia Española de Seguridad Alimentaria. Estrategia NAOS. Estrategia para la nutrición, actividad física y prevención de la obesidad. Madrid: Ministerio de Sanidad y Consumo; 2005.

3. Childhood overweight and obesity, 2011. En: Organización Mundial de la Salud [en línea] [consultado el 16/11/2015]. Disponible en www.who.int/ dietphysicalactivity/childhood/en/

4. WHO-led Commission on Ending Childhood Obesity meets o develop global responses to obesity epidemic. Second meeting of the Commission on Ending Childhood Obesity (ECHO), 13-14 January 2015. En: Organización Mundial de la Salud [en línea] [consultado el 16/11/2015]. Disponible en www.who.int/endchidhood-obesity/news/echo-second-meeting/en/ dad de Pediatría de Galicia (SOPEGA), celebrado los días 25 y 26 de octubre de 2013 en Vigo.

\section{ABREVIATURAS}

FO: Fundación Orbegozo • IC 95: intervalo de confianza del 95\% - IMC: índice de masa corporal • IOTF: Internacional Obesity Task Force • OMS: Organización Mundial de la Salud - SERGAS: Servicio Galego de Saúde.

\section{AGRADECIMIENTOS}

A la enfermería pediátrica de los centros de salud en los que se ha desarrollado este estudio, por su contribución en la realización de los controles de salud establecidos en el Programa do Neno San.

5. Moreno LA, Rodríguez G. Dietary risk factors for development of childhood obesity. Curr Opin Clin Nutr Metab Care. 2007;10:336-41.

6. Kelly AS, Barlow SE, Rao G. Severe obesity in children and adolescents: identification, associated health risks, and treatment approaches. Circulation. 2013; 128:1689-712.

7. Wang LY, Chyen D, Lee S, Lowry R. The association between body mass index in adolescence and obesity in adulthood. J Adolesc Health. 2008;42:512-8.

8. Actividades preventivas en Pediatría. Guía de apoio para o programa do Neno San. División de Asistencia Sanitaria. Consellería de Sanidade (SERGAS). Xunta de Galicia; 2004.

9. Sobradillo B, Aguirre A, Aresti U, Bilbao A, FernándezRamos C, Lizárraga A, et al. Curvas y tablas de crecimiento. Estudios longitudinal y transversal. Fundación Orbegozo. En: Patrones de crecimiento y desarrollo en España. Atlas de gráficas y tablas. Madrid: Ergón; 2004. pp. 1465-68. 
10. Aranceta Bartrina J, Serra Majem L, Foz Sala M, Moreno Esteban B. Prevalencia de obesidad en España. Med Clin (Barc). 2005;125:460-6.

11. Bueno M, Grupo PAIDOS 84. Estudio epidemiológico sobre nutrición y obesidad infantil. Proyecto universitario. Madrid: DANONE; 1985.

12. Serra Majem L, Ribas Barba L, Aranceta Bartrina J. Obesidad infantil y juvenil en España. Resultados del Estudio enKid (1988-2000). Med Clin (Barc). 2003; 121:725-32.

13. Valdés Pizarro J, Royo-Bordonada MA. Prevalence of childhood obesity in Spain: National Health Survery 2006-2007. Nutr Hosp. 2012;27:154-60.

14. Henríquez P, Doreste J, Laínez P, Estévez MD, Iglesias M. Prevalencia de obesidad y sobrepeso en adolescentes canarios. Relación con el desayuno y la actividad física. Med Clin (Barc). 2008;130:606-10.

15. Durá Trave T, Hualde Olascoaga J, Garralda Torres I. Exceso de peso corporal infantil en Navarra y su relación con la adolescencia. Med Clin (Barc). 2012; 138:52-6.

16. Posso M, Brugulat Guiteras P, Puig T, Mompart Penina A. Prevalencia y condicionantes de la obesidad en la población infantojuvenil de Cataluña, 2006-2012. Med Clin (Barc). 2014;143:475-83.

17. Sánchez Cruz J, Jiménez Moleón J, Fernández Quesada M, Sánchez M. Prevalencia de obesidad infantil y juvenil en España en 2012. Rev Esp Cardiol. 2013;66:371-6.

18. Estudio Aladino 2013. Estudio de Vigilancia del Crecimiento, Alimentación, Actividad Física,
Desarrollo Infantil y Obesidad 2013. Agencia Española de Consumo, Seguridad Alimentaria y Nutrición. Madrid: Ministerio de Sanidad, Servicios Sociales e Igualdad; 2014.

19. Park MH, Falconer C, Viner RM, Kinra S. The impact of childhood obesity on morbidity and mortality in adulthood: a systematic review. Obes Rev. 2012; 13:985-1000.

20. Sánchez M. Aspectos epidemiológicos de la obesidad infantil. Rev Pediatr Aten Primaria. Supl. 2012;(21):9-14.

21. Fundación Faustino Orbegozo Eizaguirre-Instituto de Investigación sobre Crecimiento y Desarrollo [en línea] [consultado el 16/11/2015]. Disponible en www.aepap.org/pdf/f_orbegozo_04.pdf

22. Cole TJ, Bellizzi MC, Flegal KM, Diez WH. Body mass index cut offs to define thinness in children and adolescents: international survey. BMJ. 2007;335:194-7.

23. Child growth standards: methods and development. En: Organización Mundial de la Salud [en línea] [consultado el 16/11/2015]. Disponible en www.who. int/childgrowth/standards/technical_report/en/

24. Barroso Espadero D. ¿Cuál es la mejor evidencia disponible para recomendar el tratamiento de la obesidad en niños por debajo de los cinco años de edad? Evid Pediatr. 2009;5:74.

25. Martínez Álvarez JR, Villarino Marín A, García Alcón RM, Calle Purón ME, Marrodán Serrano MD. Obesidad infantil en España: hasta qué punto es un problema de salud pública o sobre la fiabilidad de las encuestas. Nutr Clín Diet Hosp. 2013;33:80-8.

\section{CORRECCIÓN DE ERRORES}

En el editorial del número 66 (junio 2015) titulado "Antivacunas: un reto para el pediatra” (M. Aparicio Rodrigo) se han detectado varios errores en la numeración de las referencias bibliográficas en el texto. En la versión actual en la web (y el artículo descargable) se han corregido los errores. 